
the finite element method

RAIRO - Analyse numérique, tome 15, no 3 (1981), p. 265-286.

<http://www.numdam.org/item?id=M2AN_1981_15_3_265_0>

(C) AFCET, 1981, tous droits réservés.

L'accès aux archives de la revue «RAIRO - Analyse numérique » implique l'accord avec les conditions générales d'utilisation (http://www.numdam.org/ legal.php). Toute utilisation commerciale ou impression systématique est constitutive d'une infraction pénale. Toute copie ou impression de ce fichier doit contenir la présente mention de copyright.

\title{
Numdam
}

Article numérisé dans le cadre du programme

Numérisation de documents anciens mathématiques

http://www.numdam.org/ 


\title{
DISCRETE FORMS OF FRIEDRICHS' INEQUALITIES IN THE FINITE ELEMENT METHOD $\left({ }^{*}\right)$
}

\author{
by Alexander ŽENíšEK (1) \\ Communicated by P G Ciarlet
}

\begin{abstract}
Auxiliary theorems allowing to extend the theory of curved finite elements introduced in [2], [3] to the case of boundary value problems with various stable and unstable boundary condrtions are proved As an example the problem of bending of thin elastic plates is considered
\end{abstract}

Résumé - On démontre des théorèmes auxllaares quı permettent d'étendre la théorle des éléments finis courbes présentée dans [2], [3] au cas de problèmes aux limites avec des conditions aux limites stables et instables diverses Comme exemple, on présente le problème de la flexıon élastıque des plaques minces

Let $\Omega_{h}$ be a finite element approximation of a given domain $\Omega$ and $W_{h}^{s}$ a finite element subspace of the space $C^{s-1}\left(\Omega_{h}\right)$ of all functions defined on $\Omega_{h}$ which have continuous derivatives up to order $s-1$ on $\Omega_{h}(s \geqslant 1)$. The main aim of this paper is to show that for $h<\tilde{h}$ (where $\tilde{h}$ is sufficiently small) the constants $K\left(\Omega_{h}\right)$ appearing in Friedrichs' inequality and related inequalities written for functions from $W_{h}^{s}$ can be substituted by constants independent on $h$. This result allows to extend the theory of curved finite elements developed by Ciarlet and Raviart [2] and Clarlet [3] to the case of boundary value problems with various stable and unstable boundary conditions.

The inequalities appearıng in thıs paper are called discrete forms of Friedrichs' inequalities because they are written only for the functions from the finite dimensional spaces $W_{h}^{s}$.

As usual, the symbol $H^{k}(\Omega)$ will denote the Sobolev space

$$
H^{k}(\Omega)=\left\{v \in L_{2}(\Omega): D^{\alpha} v \in L_{2}(\Omega) \quad \forall|\alpha| \leqslant k\right\}
$$

(*) Reçu le 5 maı 1980

( ${ }^{1}$ ) Computıng Center of the Technical University, Obránců mıru 21, 60200

Brno, Czechoslovakıa

R A I R O Analyse numérique/Numerical Analysıs, 0399-0516/1981/265/S 500

(C) Bordas-Dunod 
where $D^{\alpha} v$ is the multiindex notation for derivatives, i.e., if $\alpha=\left(\alpha_{1}, \alpha_{2}\right) \in N^{2}$ then

$$
|\alpha|=\alpha_{1}+\alpha_{2}, \quad D^{\alpha} v=\partial^{|\alpha|} v / \partial x^{\alpha_{1}} \partial y^{\alpha_{2}} .
$$

The norm and seminorms are defined in $H^{k}(\Omega)$ by

$$
\|v\|_{k, \Omega}^{2}=\sum_{j=0}^{k}|v|_{j, \Omega}^{2}, \quad|v|_{j, \Omega}^{2}=\sum_{|\alpha|=j} \iint_{\Omega}\left(D^{\alpha} v\right)^{2} d x d y .
$$

\section{SOME DEFINITION AND LEMMAS}

Let $\Omega$ be a bounded domain in the $x, y$-plane with a sufficiently smooth boundary $\Gamma$. Let

$$
x=\varphi(s), \quad y=\psi(s), \quad a \leqslant s \leqslant b
$$

be a parametric representation of $\Gamma$. Let us triangulate the domain $\Omega$, i.e., let us divide it into a finite number of triangles (the sides of which can be curved) in such a way that two arbitrary triangles are either disjoint, or have a common vertex, or a common side. Let every triangulation $\tau$ have the property that each interior triangle (i.e. a triangle having at most one point common with the boundary) has straight sides and each boundary triangle has at most one curved side. The curved triangles of the trianguilation $\tau$ will be called ideal curved triangles.

With every triangulation $\tau$ we associate three parameters $h, \bar{h}$ and $\theta$ defined by

$$
h=\max _{T \in \tau} h_{T}, \quad \bar{h}=\min _{T \in \tau} h_{T}, \quad \theta=\min _{T \in \tau} \theta_{T}
$$

where $h_{T}$ and $\theta_{T}$ are the length of the greatest side and the smallest angle, respectively, of the triangle with straight sides which has the same vertices as the triangle $T$. We restrict ourselves to triangulations $\tau$ satisfying

$$
\begin{array}{ll}
\theta \geqslant \theta_{0}, & \theta_{0}=\text { const }>0, \\
\bar{h} \geqslant c_{0} h, & c_{0}=\text { const }>0 .
\end{array}
$$

Let $T \in \tau$ be an ideal curved triangle and $P_{1}, P_{2}, P_{3}$ a local notation of its vertices. Let $P_{2}\left(x_{2}, y_{2}\right), P_{3}\left(x_{3}, y_{3}\right)$ be the end points of the curved side of $T$ and let the symbols $s_{2}, s_{3}$ denote the values of the parameter $s$ for which

$$
x_{k}=\varphi\left(s_{k}\right), \quad y_{k}=\psi\left(s_{k}\right) \quad(k=2,3) .
$$


According to (1) and (5), the parametric representation of the curved side $P_{2} P_{3}$ of $T$ can be written in the form

$$
x=\bar{\varphi}(t) \equiv \varphi\left(s_{2}+\bar{s}_{32} t\right), \quad y=\bar{\psi}(t) \equiv \psi\left(s_{2}+\bar{s}_{32} t\right)
$$

where $0 \leqslant t \leqslant 1$ and $\bar{s}_{32}=s_{3}-s_{2}$.

Let $\varphi^{*}(t)$ and $\psi^{*}(t)$ be polynomials of degree at most $n$ which satisfy

$$
\varphi^{*}(0)=x_{2}, \quad \varphi^{*}(1)=x_{3}, \quad \psi^{*}(0)=y_{2}, \quad \psi^{*}(1)=y_{3} .
$$

Then

$$
\begin{aligned}
& \varphi^{*}(t)=x_{2}+\bar{x}_{32} t+t(1-t) p_{1}(t), \\
& \psi^{*}(t)=y_{2}+\bar{y}_{32} t+t(1-t) p_{2}(t),
\end{aligned}
$$

where $\bar{x}_{32}=x_{3}-x_{2}, \bar{y}_{32}=y_{3}-y_{2}$. The polynomials $p_{1}(t), p_{2}(t)$ depend on the form of approximation of the curved side $P_{2} P_{3}$. If $n=1$ then $p_{i}=0$ $(i=1,2)$. If $n=2$ then we require $\varphi^{*}(1 / 2)=\bar{\varphi}(1 / 2), \psi^{*}(1 / 2)=\bar{\psi}(1 / 2)$ and obtain $p_{1}=4 \bar{\varphi}(1 / 2)-2 x_{2}-2 x_{3}, p_{2}=4 \bar{\psi}(1 / 2)-2 y_{2}-2 y_{3}$. If $n>2$ then we restrict ourselves to the case $n=2 r+1(r \geqslant 1)$ and require $\varphi^{*}(t)$, $\psi^{*}(t)$ to be Hermite interpolation polynomials of functions $\bar{\varphi}(t), \bar{\psi}(t)$ uniquely determined by the function values and all derivatives up to order $r$ inclusively at the points $t_{2}=0, t_{3}=1$. This leads, with respect to (7), to the following additional conditions for $\varphi^{*}(t)$ and $\psi^{*}(t)$ :

$$
\begin{aligned}
\bar{s}_{32}^{k} \varphi^{(k)}\left(s_{i}\right) & =\varphi^{*(k)}\left(t_{i}\right), & k & =1, \ldots, r ; i=2,3, \\
\bar{s}_{32}^{k} \psi^{(k)}\left(s_{i}\right) & =\psi^{*(k)}\left(t_{i}\right), & k & =1, \ldots, r ; i=2,3 .
\end{aligned}
$$

Let $n$ be chosen. Then the approximation $T^{*}$ of the ideal curved triangle $T \in \tau$ is defined in the following way : $T^{*}$ is the triangle which has the same vertices $P_{1}, P_{2}, P_{3}$ as $T$, straight sides $P_{1} P_{2}, P_{1} P_{3}$ and the curved side defined by the equations

$$
x=\varphi^{*}(t), \quad y=\psi^{*}(t), \quad 0 \leqslant t \leqslant 1 .
$$

The curved triangle $T^{*}$ can be expressed as an image of the standard triangle $T_{0}$ which lies in the plane $\xi, \eta$ and has the vertices $R_{1}(0,0), R_{2}(1,0), R_{3}(0,1)$. The corresponding mapping is of the form

$$
\begin{aligned}
& x=x^{*}(\xi, \eta) \equiv x_{1}+\bar{x}_{2} \xi+\bar{x}_{3} \eta+\xi \eta p_{1}(\eta), \\
& y=y^{*}(\xi, \eta) \equiv y_{1}+\bar{y}_{2} \xi+\bar{y}_{3} \eta+\xi \eta p_{2}(\eta)
\end{aligned}
$$

where $\bar{x}_{k}=x_{k}-x_{1}, \bar{y}_{k}=y_{k}-y_{1}$ (see [8], [9]). 
In this paper, besides the standard triangle $T_{0}$ we shall need the triangle $T_{0}^{q}$ which lies in the plane $\xi, \eta$ and has the vertices $R_{1}(0,0), R_{2}^{q}(1+q, 0)$, $R_{3}^{q}(0,1+q), q$ being a given number, $q \geqslant 0$. The image of $T_{0}^{q}$ in transformation (13) will be denoted by $T^{* q}$. The properties of transformation (13) are summarized in lemma 1. (In this lemma and in what follows the symbol $\bar{T}$ denotes a closed triangle and the symbol $T$ its interior.)

LEMMA 1 : Let $n$ be given, let the boundary $\Gamma$ of the domain $\Omega$ be of class $C^{n+1}$ and let $q=0\left(h^{1 / 2}\right), q \geqslant 0$. If $h$ is sufficiently small then transformation (13) has the following properties:

1. The Jacobian $J^{*}(\xi, \eta)$ of transformation (13) is different from zero on $\bar{T}_{0}^{q}$.

2. The vertex $R_{1}$ is mapped onto the point $P_{1}\left(x_{1}, y_{1}\right)$, the vertex $R_{2}^{q}$ onto the point $P_{2}^{q}\left(x_{2}+\bar{x}_{2} q, y_{2}+\bar{y}_{2} q\right)$ and the vertex $R_{3}^{q}$ onto the point

$$
P_{3}^{q}\left(x_{3}+\bar{x}_{3} q, y_{3}+\bar{y}_{3} q\right) \text {. }
$$

The sides $R_{1} R_{2}^{q}$ and $R_{1} R_{3}^{q}$ are linearly mapped onto the segments $P_{1} P_{2}^{q}$ and $P_{1} P_{3}^{q}$, respectively, and the side $R_{2}^{q} R_{3}^{q}$ is mapped onto the arc s $s^{* q}=P_{2}^{q} P_{3}^{q}$ which has the parametric equations

$$
\begin{aligned}
& x=x_{2}+\bar{x}_{2} q+\bar{x}_{32} \eta+(1+q-\eta) \eta p_{1}(\eta), \\
& y=y_{2}+\bar{y}_{2} q+\bar{y}_{32} \eta+(1+q-\eta) \eta p_{2}(\eta),
\end{aligned}
$$

where $0 \leqslant \eta \leqslant 1+q$.

3. The mapping (13) maps the triangle $\bar{T}_{0}^{q}$ one-to-one onto the triangle $\bar{T}^{* q}$ whose boundary $\partial T^{* q}$ is the union of the segments $P_{1} P_{2}^{q}, P_{1} P_{3}^{q}$ and the arc $s^{* q}$.

4. Both the mapping (13) and its inverse mapping are of class $C^{\infty}$ and it holds for $(\xi, \eta) \in \bar{T}_{0}^{q},(x, y) \in \bar{T}^{* q}$ :

$$
\begin{gathered}
c_{1} h_{T}^{2} \leqslant\left|J^{*}(\xi, \eta)\right| \leqslant c_{2} h_{T}^{2}, \quad c_{\imath}=\text { const }>0, \\
D^{\alpha} x^{*}(\xi, \eta)=0\left(h_{T}^{|\alpha|}\right), \quad D^{\alpha} y^{*}(\xi, \eta)=0\left(h_{T}^{|\alpha|}\right), \quad|\alpha|=1,2, \ldots, \\
D^{\alpha} \xi^{*}(x, y)=0\left(h_{T}^{-1}\right), D^{\alpha} \eta^{*}(x, y)=0\left(h_{T}^{-1}\right), \quad|\alpha|=1,2, \ldots,
\end{gathered}
$$

where

$$
\xi=\xi^{*}(x, y), \quad \eta=\eta^{*}(x, y)
$$

is the inverse mapping to the mapping (13).

5. Let $\widetilde{S}_{1}, \widetilde{S}_{2}$ be two arbitrary points of the triangle $\bar{T}_{0}^{q}$ and $S_{1}, S_{2}$ their images 
with respect to the mapping (13). Let $\varepsilon$ be the distance between $\widetilde{S}_{1}, \widetilde{S}_{2}$ and $\delta$ the distance between $S_{1}, S_{2}$. Then

$$
b_{1} \varepsilon h_{T} \leqslant \delta \leqslant b_{2} \varepsilon h_{T}, \quad b_{1} \varepsilon h \leqslant \delta \leqslant b_{2} \varepsilon h
$$

where $b_{1}>0, b_{2}>0$ are constants independent on $h_{T}$ and $\varepsilon$.

The proof of lemma 1 is a straightforward generalization of considerations introduced in [7], [8] and [9]. Thus it is omitted.

Let us replace the ideal curved triangles $\bar{T}$ of the triangulation $\tau$ of $\Omega$ by their approximations $\bar{T}^{*}$ defined above and denote such a changed triangulation by $\tau_{h}$. The union of the closed triangles of $\tau_{h}$ will be denoted by $\bar{\Omega}_{h}$ and the boundary of $\Omega_{h}$ by $\Gamma_{h}$. Using triangular finite $C^{m}$-elements we shall construct finite dimensional subspaces $W_{h}^{m+1}$ of $C^{m}\left(\Omega_{h}\right)$. We restrict ourselves to the cases $m=0$ and $m=1$. As such constructions are well-known (see, e.g., [7], [8], [5], [9]) we introduce only the properties necessary for our considerations.

Let us start with $m=0$. If $n=1$ then $\Omega_{h}$ is a polygonal domain and the restriction $\left.v\right|_{\bar{T}}$ of $v \in W_{h}^{1}$ to an arbitrary triangle $\bar{T} \in \tau_{h}$ is a linear function uniquely determined by function values prescribed at the vertices of $\bar{T}$.

If $n=2$ then $\Gamma_{h}$ is piecewise quadratic and the restriction $\left.v\right|_{T}$ of $v \in W_{h}^{1}$ to an arbitrary interior triangle is a quadratic polynomial uniquely determined by the function values $v\left(P_{i}\right), v\left(Q_{j k}\right)(i=1,2,3 ; j=1,2 ; k=2,3 ; j<k)$ where $P_{i}$ are the vertices of $\bar{T}$ and $Q_{j k}$ the mid-points of the sides $P_{j} P_{k}$. The restriction $\left.v\right|_{\bar{T}^{*}}$ of $v \in W_{h}^{1}$ to an arbitrary boundary triangle $\bar{T}^{*} \in \tau_{h}$ is such a function that

$$
p(\xi, \eta)=\left.v\right|_{\bar{T}} ^{*}\left(x^{*}(\xi, \eta), y^{*}(\xi, \eta)\right)
$$

is a quadratic polynomial uniquely determined by the function values $p\left(R_{i}\right)=\left.v\right|_{\bar{T}^{*}}\left(P_{i}\right), p\left(S_{j k}\right)=\left.v\right|_{\bar{T}^{*}}\left(Q_{j k}\right)$ where $S_{j k}$ is the midpoint of $R_{j} R_{k}$. (Let us note that $Q_{23}=\left(\varphi^{*}(1 / 2), \psi^{*}(1 / 2)\right)$.)

If $n=2 k+1(k \geqslant 1)$ then $\Gamma_{h}$ is piecewise of degree $2 k+1$ and the restriction $\left.v\right|_{\bar{T}}$ of $v \in W_{h}^{1}$ to an arbitrary interior triangle $\bar{T} \in \tau_{h}$ is a polynomial of degree $2 k+1$ uniquely determined by the parameters

$$
D^{\alpha} v\left(P_{i}\right),|\alpha| \leqslant k \quad(i=1,2,3) ; \quad D^{\alpha} v\left(P_{0}\right), \quad|\alpha| \leqslant k-1
$$

where $P_{i}$ are vertices and $P_{0}$ the centre of gravity of $\bar{T}$. The restriction $\left.v\right|_{\bar{T}^{*}}$ of $v \in W_{h}^{1}$ to an arbitrary boundary triangle $\bar{T}^{*} \in \tau_{h}$ is such a function that the function $p(\xi, \eta)$ defined by (19) is a polynomial of degree $2 k+1$ uniquely determined by the parameters

$$
D^{\alpha} p\left(R_{i}\right), \quad|\alpha| \leqslant k \quad(i=1,2,3) ; \quad D^{\alpha} p\left(R_{0}\right), \quad|\alpha| \leqslant k-1
$$


where $R_{0}$ is the centre of gravity of $T_{0}$. Each of the derivatives $D^{\alpha} p\left(R_{k}\right)$, $|\alpha|=j$ is a linear combination of the derivatives $D^{\alpha} v\left(P_{k}\right),|\alpha|=j$ and can be obtained from (19) by means of the rule of differentiation of a composite function. (Let us note that $P_{0}$ is in this case the image of $R_{0}$ in transformation (13).)

In the case $m=1$ we restrict ourselves to the case of the simplest $C^{1}$-elements. Thus the restriction $\left.v\right|_{\bar{T}}$ of $v \in W_{h}^{2}$ to an arbitrary interior triangle $\bar{T} \in \tau_{h}$ is Bell's polynomial of fifth degree uniquely determined by the parameters

$$
D^{\alpha} v\left(P_{i}\right), \quad|\alpha| \leqslant 2, \quad i=1,2,3
$$

and by the condition that $\partial v / \partial v_{j k}$ be a polynomial of third degree along the side $P_{j} P_{k}(j<k, j=1,2 ; k=2,3), v_{j k}$ being the normal to $P_{j} P_{k}$. The restriction $\left.v\right|_{T^{*}}$ of $v \in W_{h}^{2}$ to an arbitrary boundary triangle $\bar{T}^{*} \in \tau_{h}$ is such a function that the function $p(\xi, \eta)$ defined by (19) is a polynomial of degree $4+n$ uniquely determined by the parameters described in [9, theorem 3]. The number $n$ depends usually on the boundary conditions prescribed on $\Gamma$.

Let us consider the case that $\Omega$ represents the middle plane of a thin elastic plate. Then from the point of view of boundary conditions the boundary $\Gamma$ is divided into three disjoint parts : a clamped part $\Gamma_{1}$, a simply supported part $\Gamma_{2}$ and a free part $\Gamma_{3}$. Let $\Gamma_{h i}(i=1,2,3)$ be the part of $\Gamma_{h}$ approximating $\Gamma_{i}$ and let $n_{i}$ be the degree of curved sides $c_{h}$ from which $\Gamma_{h i}$ consists. It is not difficult to see that the degrees $n_{1}=3, n_{2}=5$ are necessary and sufficient for the validity of implication (98). Further, it will be seen in section 3 that the degrees $n_{1}=3, n_{2}=5, n_{3}=3$ are sufficient for the validity of inequality (99). In this case also the second term on the right-hand side of inequality $(100)$ is of the same numerical accuracy as the first term. Thus we set

$$
n_{1}=3, n_{2}=5, n_{3}=3 \text {. }
$$

The following lemma will be useful in our considerations :

Lemma 2 : Let a part $\Delta \Gamma$ of the boundary $\Gamma$ be of class $C^{n+1}$ and let $c \subset \Delta \Gamma$ be the curved side of an ideal curved triangle. Let (6) be parametric equations of $c$. Let the functions $\varphi^{*}(t), \psi^{*}(t)$ defining the approximation $c_{h}$ of $c$ be either Lagrange or Hermite interpolation polynomials of degree $n$ of the functions $\bar{\varphi}(t), \bar{\psi}(t)$ on the interval $I=[0,1]$. Then it holds on $I$

$$
\begin{gathered}
\left|\varphi^{*(j)}(t)-\bar{\varphi}^{(j)}(t)\right| \leqslant C h_{\mathrm{T}}^{n+1}, \quad j=0,1, \ldots, n, \\
\left|\psi^{*(j)}(t)-\bar{\psi}^{(j)}(t)\right| \leqslant C h_{T}^{n+1}, \quad j=0,1, \ldots, n, \\
\bar{\rho}(t) \geqslant C h_{T}
\end{gathered}
$$

R.A.I.R.O. Analyse numérique/Numerical Analysis 
where

$$
\bar{\rho}(t)=\left\{\left[\bar{\varphi}^{\prime}(t)\right]^{2}+\left[\bar{\psi}^{\prime}(t)\right]^{2}\right\}^{1 / 2}
$$

and where $C$ depends only on $\Gamma$.

The proof of lemma 2 is similar to the proof of [10, lemma 2]. Thus it is omitted.

At the end of this section we introduce a notion of a natural extension $\bar{v}$ of a function $v \in W_{h}^{m+1}$ from the domain $\Omega_{h}$ to the domain $\Omega_{h}+\left(\Omega-\Omega_{h}\right)$. This notion will be important in the proof of theorems 1 and 2 .

The set $\Omega-\Omega_{h}$ is a union of sets $T_{J}-T_{J}^{*}(j=1, \ldots, N)$ where $N$ is the number of curved triangles in $\tau, \bar{T}_{j} \in \tau$ is an ideal curved triangle and $\bar{T}_{J}^{*}$ its approximation. Let us choose $q=0\left(h^{1 / 2}\right)$ and let $\bar{T}_{j}^{* q}$ be the image of $\bar{T}_{0}^{q}$ in transformation (13). (The index $j$ expresses that transformation (13) concerns the triangle $\bar{T}_{J}$ ) If $T_{J}-T_{J}^{*} \neq \varnothing$ then, according to (18) and (24), the part of the curved side of $T_{J}$ which forms a part of the boundary of $T_{J}-T_{J}^{*}$ lies in $T_{J}^{* q}-T_{J}^{*}$.

If we transform the restriction $\left.v\right|_{T_{j}^{*}}$ of $v \in W_{h}^{m+1}$ from $\bar{T}_{J}^{*}$ onto the standard triangle $\bar{T}_{0}$ by means of transformation (13), we obtain a polynomial $p_{\jmath}(\xi, \eta)$ of degree $d(d=n$ if $m=0, d=4+n$ if $m=1)$,

$$
p_{J}(\xi, \eta)=\left.v\right|_{\bar{T}_{j}^{*}}\left(x_{J}^{*}(\xi, \eta), y_{j}^{*}(\xi, \eta)\right) .
$$

The polynomial (27) is defined uniquely in the whole $\xi$, $\eta$-plane. Transforming $p_{f}(\xi, \eta)$ from the triangle $\bar{T}_{0}^{q}$ onto the triangle $\bar{T}_{J}^{* q}$ by means of the inverse transformation $(17)$, we obtain a function $v^{q}(x, y)$. Its restriction to the domain $T_{J}^{*}+\left(T_{J}-T_{J}^{*}\right)$ is the natural extension of $\left.v\right|_{T_{J}^{*}}$ from $T_{J}^{*}$ to $T_{J}+\left(T_{J}-T_{J}^{*}\right)$. Doing this for all boundary triangles we obtain the natural extension $\bar{v}$ of $v \in W_{h}^{m+1}$ from $\Omega_{h}$ to $\Omega_{h}+\left(\Omega-\Omega_{h}\right)$.

\section{DISCRETE FORMS OF FRIEDRICHS' INEQUALITIES}

THEOREM $1:$ Let $n=2 k+1(k \geqslant 0)$ or $n=2$. Let the boundary $\Gamma$ of $\Omega$ be of class $C^{n+1}$. Let $S$ be a part of $\Gamma$ consisting of a finite number of disjoint arcs $S_{J} \subset \Gamma$ such that mes $S_{J} \neq 0$. Let $S_{h}$ be the part of $\Gamma_{h}$ which approximates $S$. Let every triangulation $\tau$ be chosen in such a way that $S$ is a union of some sides of ideal boundary triangles. Then the constant $K\left(\Omega_{h}\right)$ appearing in the inequality

$$
\|v\|_{1, \Omega_{h}}^{2} \leqslant K\left(\Omega_{h}\right)\left[\int_{S_{h}} v^{2} d s+|v|_{1, \Omega_{h}}^{2}\right] \quad \forall v \in W_{h}^{1}
$$


can be chosen in such a way that

$$
K\left(\Omega_{h}\right) \rightarrow K_{1}(\Omega) \quad \text { if } \quad h \rightarrow 0
$$

where $K_{1}(\Omega)$ is an arbitrary constant which can occur in Friedrichs' inequality

$$
\|v\|_{1, \Omega}^{2} \leqslant K_{1}(\Omega)\left[\int_{S} v^{2} d s+|v|_{1, \Omega}^{2}\right] \quad \forall v \in H^{1}(\Omega) .
$$

THEOREM 2 : Let $n \geqslant 3$ and let the other assumptions of theorem 1 be satisfied. Let $S$ be not a part of a straight line. Then the constant $K\left(\Omega_{h}\right)$ appearing in the inequality

$$
\|v\|_{2, \Omega_{h}}^{2} \leqslant K\left(\Omega_{h}\right)\left[\int_{S_{h}} v^{2} d s+|v|_{2, \Omega_{h}}^{2}\right] \quad \forall v \in W_{h}^{2}
$$

can be chosen in such a way that

$$
K\left(\Omega_{h}\right) \rightarrow K_{2}(\Omega) \quad \text { if } \quad h \rightarrow 0
$$

where $K_{2}(\Omega)$ is an arbitrary constant which can occur in the inequality

$$
\|v\|_{2, \Omega}^{2} \leqslant K_{2}(\Omega)\left[\int_{S} v^{2} d s+|v|_{2, \Omega}^{2}\right] \quad \forall v \in H^{2}(\Omega) .
$$

Proof of theorems 1 and $2:$ If $v \equiv 0$ then both (28) and (31) hold with an arbitrary constant $K\left(\Omega_{h}\right)$. Thus we consider only $v \not \equiv 0$. Let us set

$$
\omega_{h+}=\Omega_{h}-\Omega, \quad \omega_{h-}=\Omega-\Omega_{h} .
$$

It holds

$$
\frac{\int_{S_{h}} v^{2} d s+|v|_{i, \Omega_{h}}^{2}}{\|v\|_{i, \Omega_{h}}^{2}}=\frac{\left(\int_{S} \bar{v}^{2} d s+|\bar{v}|_{i, \Omega}^{2}\right)\left(1+\varepsilon_{i, \Gamma}+\varepsilon_{i+}-\varepsilon_{i-}\right)}{\|\bar{v}\|_{i, \Omega}^{2}\left(1+\delta_{i+}-\delta_{i-}\right)}
$$

where

$$
\begin{gathered}
\varepsilon_{i \pm}=|\bar{v}|_{i, \omega_{h \pm}}^{2} /\left(\int_{S} \bar{v}^{2} d s+|\bar{v}|_{i, \Omega}^{2}\right), \\
\varepsilon_{i, \Gamma}=\left(\int_{S_{h}} v^{2} d s-\int_{S} \bar{v}^{2} d s\right) /\left(|\bar{v}|_{i, \Omega}^{2}+\int_{S} \bar{v}^{2} d s\right), \\
\delta_{i \pm}=\|\bar{v}\|_{i, \omega_{h \pm}}^{2} /\|\bar{v}\|_{i, \Omega}^{2} .
\end{gathered}
$$


Taking into account (30) and (33) we see that it suffices to prove

$$
\varepsilon_{i, \Gamma} \rightarrow 0, \varepsilon_{i \pm} \rightarrow 0, \delta_{i \pm} \rightarrow 0 \text { if } h \rightarrow 0(i=1,2) .
$$

a) First we shall prove that

$$
|\bar{v}|_{0, \Omega}^{2} \geqslant C h^{2} A\left(\hat{\alpha}_{i}^{j}\right), \quad|\bar{v}|_{1, \Omega}^{2} \geqslant C B\left(\hat{\alpha}_{i}^{j}\right)
$$

where $C$ is an absolute constant and

$$
\begin{gathered}
A\left(\hat{\alpha}_{i}^{j}\right)=\sum_{j=1}^{N} \sum_{i=1}^{d}\left(\hat{\alpha}_{i}^{j}\right)^{2}, \\
B\left(\hat{\alpha}_{i}^{j}\right)=\sum_{j=1}^{N}\left[\sum_{i=1}^{s}\left(\hat{\alpha}_{i}^{j}-\hat{\alpha}_{0}^{j}\right)^{2}+\sum_{i=s+1}^{d}\left(\hat{\alpha}_{i}^{j}\right)^{2}\right], \\
\hat{\alpha}_{0}^{j}=\left(\hat{\alpha}_{1}^{j}+\hat{\alpha}_{2}^{j}+\cdots+\hat{\alpha}_{s}^{j}\right) / s .
\end{gathered}
$$

The symbols appearing on the right-hand sides of (40)-(42) have the following meaning : $N$ is the number of the boundary triangles and $\hat{\alpha}_{i}^{j}$ are the parameters uniquely determining the polynomial $p_{j}(\xi, \eta)$ which is defined by (27) and which can be written in the form

$$
p_{j}(\xi, \eta)=\sum_{i=1}^{d} \hat{\alpha}_{i}^{j} \hat{b}_{i}(\xi, \eta)
$$

where $\hat{b}_{i}(\xi, \eta)$ are basis functions corresponding to the parameters $\hat{\alpha}_{i}$. In the case of $C^{0}$-elements the number of basis functions is given by

$$
d=(n+1)(n+2) / 2 \text {, }
$$

in the case of $C^{1}$-elements by $d=(n+5)(n+6) / 2$. The parameters $\hat{\alpha}_{i}$ and the basis functions $\hat{b}_{i}(\xi, \eta)$ are ordered in such a way that the first $s$ parameters $\hat{\alpha}_{1}, \ldots, \hat{\alpha}_{s}$ are all parameters which have the meaning of function values. Thus

$$
\sum_{i=1}^{s} \hat{b}_{i}(\xi, \eta)=1 .
$$

In proving the first estimate (39) we start from the inequality

$$
|\bar{v}|_{0, \Omega}^{2} \geqslant \sum_{j=1}^{N}|\bar{v}|_{0, T_{j}}^{2}
$$

where $T_{j} \subset \Omega(j=1, \ldots, N)$ are ideal boundary triangles. Let $\kappa_{j}$ be the arc which lies in the $\xi$, $\eta$-plane and which is mapped by transformation $(13)_{j}$ 
onto the arc $\partial T_{j} \cap \Gamma, \partial T_{j}$ being the boundary of $T_{j}$. Let $0<r<1 / 2$ be a fixed number. Let $T_{0}(r)$ be the triangle which lies in the $\xi$, $\eta$-plane and has the vertices $R_{1}^{r}(0,0), R_{2}^{r}(1-r, 0), R_{3}^{r}(0,1-r)$. It follows from (18) and (24) that for sufficiently small $h$ the arcs $\kappa_{j}(j=1, \ldots, N)$ lie outside the triangle $T_{0}(r)$. Thus it holds, according to (4) and (14),

$$
|\bar{v}|_{0, T_{j}}^{2} \geqslant c_{1} c_{0} h^{2}\left|p_{j}\right|_{0, T_{0}(r)}^{2}
$$

where the polynomial $p_{j}(\xi, \eta)$ is given by (43). It follows from the equivalence of norms in a finite dimensional space that

$$
\left|p_{j}\right|_{0, T_{0}(r)}^{2} \geqslant \hat{c} \sum_{i=1}^{d}\left(\hat{\alpha}_{i}^{j}\right)^{2}
$$

where the constant $\hat{c}$ depends only on $T_{0}(r)$ and on the basis functions $\hat{b}_{i}(\xi, \eta)$. Relations (45), (46) and (47) imply the first estimate (39).

Now we prove the second estimate (39). After some elementary calculations we obtain

$$
|\bar{v}|_{1, \Omega}^{2} \geqslant \sum_{j=1}^{N}|\bar{v}|_{1, T_{j}}^{2} \geqslant c_{0}^{2} c_{2}^{-1} c^{*} \sum_{j=1}^{N}\left|p_{j}\right|_{1, T_{0}(r)}^{2}
$$

where $c^{*}$ is a constant depending only on $\theta_{0}$ and where the meaning of the other symbols is the same as in the preceding case. Let $n^{*}=n$ in the case of $C^{0}$-elements and $n^{*}=n+4$ in the case of $C^{1}$-elements. The seminorm $\left|p_{j}\right|_{1, T_{0}(r)}$ is a norm of the class $\left[p_{j}\right] \in P_{n^{*}} / P_{0}$ where $P_{k}$ denotes the space of all polynomials $p(\xi, \eta)$ of degree $\leqslant k$. Thus

$$
\left|p_{j}\right|_{1, T_{0}(r)}^{2} \geqslant \hat{c} \inf _{p \in\left[p_{j}\right]_{i}} \sum_{i=1}^{d}\left(\hat{\alpha}_{i}\right)^{2}
$$

where $\hat{\alpha}_{1}, \ldots, \hat{\alpha}_{d}$ are the parameters uniquely determining the polynomial $p(\xi, \eta) \in\left[p_{j}\right]$. As every polynomial $p \in\left[p_{j}\right]$ can be written in the form $p=p_{j}+K$ with $K \in P_{0}$ it is easy to find that

$$
\inf _{p \in\left[p_{j}\right]} \sum_{i=1}^{d}\left(\hat{\alpha}_{i}\right)^{2}=\sum_{i=1}^{s}\left(\hat{\alpha}_{i}^{j}-\hat{\alpha}_{0}^{j}\right)^{2}+\sum_{i=s+1}^{d}\left(\hat{\alpha}_{i}^{j}\right)^{2}
$$

where $\hat{\alpha}_{0}^{j}$ is defined by (42). Relations (48), (49) and (50) imply the second estimate (39).

b) Now we estimate $\varepsilon_{k+}, \varepsilon_{k_{-}}, \delta_{k_{+}}, \delta_{k_{-}}(k=1,2)$. If $v=$ const on the union $U$ of the boundary triangles $\bar{T}_{j}^{*}$ then $\varepsilon_{k \pm}=0$. Let $v \neq$ const on $U$. Then $B\left(\hat{\alpha}_{i}^{j}\right)>0$ and we can write, according to (30), (33), (35) and (39),

$$
\varepsilon_{k-} \leqslant C \cdot K_{k}(\Omega)|\bar{v}|_{k, \omega_{h}-}^{2} / B\left(\hat{\alpha}_{i}^{j}\right) \quad(k=1,2)
$$


where $C$ is an absolute constant. (In what follows $C$ will denote an absolute constant not necessarily the same in any two different places.)

Let $\sigma$ be a quadrilateral lying in the $\xi$, $\eta$-plane, having vertices $A_{1}(1-\beta, 0)$, $A_{2}(1+\beta, 0), A_{3}(0,1+\beta), A_{4}(0,1-\beta)$ and containing all $\operatorname{arcs} \kappa_{j}(j=1, \ldots, N)$. According to (18) and (24), we can choose $\beta$ so small that

$$
\text { mes } \sigma=0\left(h^{n}\right) \text {. }
$$

Using (14), (16) and (44) we find

where

$$
\begin{aligned}
|\bar{v}|_{1, \omega_{h-}}^{2} & =\sum_{j=1}^{N}|\bar{v}|_{1, T_{j}-T_{j}^{*}}^{2} \leqslant C \sum_{j=1}^{N}\left|p_{j}\right|_{1, \sigma}^{2}= \\
& =C \sum_{j=1}^{N}\left|p_{j}-\hat{\alpha}_{0}^{j}\right|_{1, \sigma}^{2} \leqslant C \sum_{j=1}^{N} M_{j}^{2} \operatorname{mes} \sigma
\end{aligned}
$$

$$
M_{j}=\max \left\{\max _{i=1, \ldots, s}\left|\hat{\alpha}_{i}^{j}-\hat{\alpha}_{0}^{j}\right|, \max _{i=s+1, \ldots, d}\left|\hat{\alpha}_{i}^{j}\right|\right\} .
$$

As, according to (54) and (41),

$$
\left(\sum_{j=1}^{N} M_{j}^{2}\right) / B\left(\hat{\alpha}_{i}^{j}\right) \leqslant 1
$$

relations (51), (52) and (53) imply

$$
\varepsilon_{1-}=O\left(h^{n}\right) \text {. }
$$

In the same way we can obtain $\varepsilon_{1+}=0\left(h^{n}\right)$.

As to the estimate of $\varepsilon_{2-}$ we can prove

$$
\varepsilon_{2-}=0\left(h^{n-2}\right) .
$$

Estimate (57) follows from (51), (52), (55) and from the estimate

$$
|\bar{v}|_{2, T_{j}-T_{j}}^{2} \leqslant C\left(\left|p_{j}\right|_{2, \sigma}^{2} h^{-2}+\left|p_{j}\right|_{1, \mathrm{\sigma}}^{2}\right) \text {. }
$$

In the same way we obtain $\varepsilon_{2+}=0\left(h^{n-2}\right)$.

Now we estimate $\delta_{k+}$ and $\delta_{k-}(k=1,2)$. Let us set for the sake of brevity

$$
\begin{gathered}
F(\bar{v})=|\bar{v}|_{0, \omega_{h}-}^{2} /\left(h^{2} A\left(\hat{\alpha}_{i}^{j}\right)\right), \\
G_{k}(\bar{v})=\left(\sum_{r=1}^{k}|\bar{v}|_{r, \omega_{h^{-}}}^{2}\right) / B\left(\hat{\alpha}_{i}^{j}\right) \quad(v \neq \text { const on } U) .
\end{gathered}
$$

If $v=$ const on $U$ then, according to (37) and (39),

$$
\delta_{k-} \leqslant C . F(\bar{v}) \text {. }
$$

vol. $15, \mathrm{n}^{\circ} 3,1981$ 
If $v \neq$ const on $U$ then

$$
\delta_{k-} \leqslant C\left[F(\bar{v})+G_{k}(\bar{v})\right] .
$$

According to preceding considerations, $G_{1}(\bar{v})=0\left(h^{n}\right), G_{2}(\bar{v})=0\left(h^{n-2}\right)$. It remains to estimate $F(\bar{v})$. It is easy to find

$$
|\bar{v}|_{0, \omega_{h-}}^{2} \leqslant C h^{2}\left(\sum_{j=1}^{N} m_{j}^{2}\right) \text { mes } \sigma
$$

where

$$
m_{j}=\max _{i=1, \ldots, d}\left|\hat{\alpha}_{i}^{j}\right|
$$

As

$$
\left(\sum_{j=1}^{N} m_{j}^{2}\right) / A\left(\hat{\alpha}_{i}^{j}\right) \leqslant 1
$$

we obtain, according to (52), (59) and (61), $F(\bar{v})=0\left(h^{n}\right)$. Thus

$$
\delta_{1-}=0\left(h^{n}\right), \quad \delta_{2-}=0\left(h^{n-2}\right) .
$$

Similarly $\delta_{1+}=0\left(h^{n}\right), \delta_{2+}=0\left(h^{n-2}\right)$.

According to the assumptions of theorems 1 and 2 concerning $n$, we proved (38) for the terms $\varepsilon_{k \pm}, \delta_{k \pm}(k=1,2)$.

c) It remains to estimate $\varepsilon_{k, \Gamma}(k=1,2)$. It holds

$$
\begin{gathered}
\int_{S_{h}} v^{2} d s=\sum_{j=1}^{N_{S}} \int_{0}^{1} v^{2}\left(\varphi_{j}^{*}(t), \psi_{j}^{*}(t)\right) \rho_{j}^{*}(t) d t \\
\int_{S} \bar{v}^{2} d S=\sum_{j=1}^{N_{S}} \int_{0}^{1} \bar{v}^{2}\left(\bar{\varphi}_{j}(t), \bar{\psi}_{j}(t)\right) \bar{\rho}_{j}(t) d t
\end{gathered}
$$

where $N_{S}$ is the number of boundary triangles along $S, \bar{\rho}_{j}(t)$ is given by (26) and

$$
\rho^{*}(t)=\left\{\left[\varphi^{*^{\prime}}(t)\right]^{2}+\left[\psi^{*^{\prime}}(t)\right]^{2}\right\}^{1 / 2} .
$$

Let us set

$$
\Delta_{j 1}=\varphi_{j}^{*}(t)-\bar{\varphi}_{j}(t), \Delta_{j 2}=\psi_{j}^{*}(t)-\bar{\psi}_{j}(t) .
$$

According to lemma 2, it holds for the $i$-th derivative

$$
\Delta_{j 1}^{(i)}=0\left(h_{T_{j}}^{n+1}\right), \Delta_{j 2}^{(i)}=0\left(h_{T_{j}}^{n+1}\right) \quad(i=0,1, \ldots, n) .
$$


Using Taylor's formula we can write

$v^{2}\left(\varphi_{J}^{*}(t), \psi_{J}^{*}(t)\right)=\bar{v}^{2}\left(\bar{\varphi}_{J}(t), \bar{\psi}_{J}(t)\right)+$

$$
+2 \bar{v}\left(Q_{\jmath}\right) \frac{\partial \bar{v}}{\partial x}\left(Q_{\jmath}\right) \Delta_{\jmath 1}+2 \bar{v}\left(Q_{\jmath}\right) \frac{\partial \bar{v}}{\partial y}\left(Q_{\jmath}\right) \Delta_{\jmath 2}
$$

where

$$
Q_{J}=\left(\bar{\varphi}_{J}(t)+\theta_{J} \Delta_{J 1}, \bar{\psi}_{J}(t)+\theta_{J} \Delta_{J 2}\right), \quad 0<\theta_{J}<1 .
$$

Using (24), (25), (26) and (67) it is easy to find

$$
\rho_{J}^{*}(t)=\bar{\rho}_{J}(t)\left[1+O\left(h_{T_{J}}^{n}\right)\right]
$$

Subtracting (66) from (65) and using (69)-(71) we obtain after an easy computation in which we use the fact that both $Q_{J}$ and $\left(\bar{\varphi}_{J}(t), \bar{\psi}_{J}(t)\right)$ belong to $\bar{T}_{j}+\bar{T}_{J}^{*} \subset \bar{T}_{J}^{* q}:$

$$
\left|\int_{S_{h}} v^{2} d s-\int_{S} \bar{v}^{2} d s\right| \leqslant C h^{n} \sum_{J=1}^{N_{S}} m_{J}^{2} \operatorname{mes} c_{J} \leqslant C h^{n+1} \sum_{J=1}^{N_{S}} m_{J}^{2}
$$

where $c_{J}=\partial T_{J} \cap \Gamma$. Using (30), (33), (36), (39), (63) and (72) we obtain

$$
\varepsilon_{k, \Gamma}=0\left(h^{n-1}\right)(k=1,2) .
$$

Thus (38) is proved in the case $n \geqslant 2$.

It remains to estimate $\varepsilon_{1, \Gamma}$ in the case $n=1$. Let us denote $H_{J}=\min _{c_{J}}|\bar{v}|$ where $c_{J}=\partial T_{J} \cap \Gamma$. Then we can write

$$
|\bar{v}|_{1, \Omega}^{2}+\int_{S} \bar{v}^{2} d s \geqslant C \sum_{J=1}^{N_{S}}\left\{\sum_{i=1}^{3}\left(\hat{\alpha}_{\imath}^{J}-\hat{\alpha}_{0}^{J}\right)^{2}+h\left(H_{\jmath}\right)^{2}\right\}
$$

because mes $c_{J} \geqslant h, N_{S} \leqslant N$ and the numbers $s$ and $d$ appearing in (41) satisfy $s=d=3$. Let us choose $\gamma$ such that $0<\gamma<\frac{1}{2}$. Then after a simple analysis (which is enabled by a simple structure of finite elements in the case $n=1$ ) it can be found that there exists $h_{\gamma}$ (dependent only on $\gamma$ ) such that for $h<h_{\gamma}$ we have

$$
\sum_{J=1}^{N_{S}}\left\{\sum_{\imath=1}^{3}\left(\hat{\alpha}_{\imath}^{J}-\hat{\alpha}_{0}^{J}\right)^{2}+h\left(H_{J}\right)^{2}\right\} \geqslant \frac{1}{2} h^{2-2 \gamma} \sum_{J=1}^{N_{S}} m_{J}^{2} .
$$

Thus, according to (36), (72)-(74), we obtain $\varepsilon_{1, \Gamma}=O\left(h^{2 \gamma}\right)$. Theorems 1 and 2 are completely proved. 
Remark 1 : In order to prove theorems 1 and 2 together we restricted ourselves to one type of boundary $C^{1}$-elements in theorem 2 . Usually we use two types of boundary $C^{1}$-elements (see (23)). In such a case the assumptions of theorem 2 can be easily modified. The proof of theorem 2 remains the same, only some formulas are more complicated.

Remark 2: Under the assumption of theorem 1 that $\Gamma$ is of class $C^{n+1}$ we obtained the maximum rate of convergence in the case of $\varepsilon_{1 \pm}, \delta_{1, \pm}$ : $\varepsilon_{1 \pm}=0\left(h^{n}\right), \delta_{1, \pm}=0\left(h^{n}\right)$. The following two statements (i) and (ii) allow to weaken this assumption. (The rate of convergence will be then lower but this is not important in our considerations.)

(i) Let $n=2 k+1(k \geqslant 1)$ and $k \leqslant \bar{n} \leqslant n$. If we assume in lemma 2 that $\Gamma$ is of class $C^{\bar{n}+1}$ then

$$
\left|\varphi^{*(j)}(t)-\bar{\varphi}^{(j)}(t)\right| \leqslant C h_{T}^{n+1}, j=0,1, \ldots, \bar{n}
$$

where $\varphi^{*}(t)$ is the Hermite interpolation polynomial of degree $n$ of the function $\bar{\varphi}(t)$ (see Section 1). Relation (24b) is modified in the same way. (This statement can be proved similarly as the first part of lemma 2 ; instead of the classic remainder theorem for Hermite interpolation we use a one-dimensional analogy of $[1$, theorem 2].)

(ii) Let $n=2 k+1(k \geqslant 2)$ and $k \leqslant \bar{n}<n$. Let the right-hand sides of (13) be polynomials of degree $n$ and let us assume in lemma 1 that $\Gamma$ is of class $C^{n}+1$. Then estimates (15), (16) hold for $|\alpha|=1, \ldots, \bar{n}-1$. The remaining assertions of lemma 1 are not changed. (This statement follows from (i).)

Remark 3 : The question of weakening the assumptions of theorem 2 is quite topical. It may happen that $\Gamma$ is only of class $C^{4}$ and we must approximate the simply supported part $\Gamma_{2}$ of $\Gamma$ piecewise by quintic arcs in order to guarantee (98).

Inspecting the proof of theorems 1 and 2 and changing lemmas 1 and 2 according to (ii) and (i), respectively, we obtain the following corollaries :

COROLlary $1:$ Let $n=2 k+1(k \geqslant 0)$ or $n=2$ and let $W_{h}^{1}$ be the corresponding $C^{0}$-finite element space which is described in section 1 . Let the boundary $\Gamma$ of $\Omega$ be of class $C^{\bar{n}+1}$ where $\bar{n}=n$ for $n \leqslant 3$ and $k+1 \leqslant \bar{n} \leqslant n$ for

$$
n=2 k+1(k \geqslant 2) \text {. }
$$

Let the assumptions of theorem 1 concerning $S$ and $S_{h}$ be satisfied. Then

$$
\|v\|_{1, \Omega_{h}}^{2} \leqslant C_{1}\left[\int_{S_{h}} v^{2} d s+|v|_{1, \Omega_{h}}^{2}\right] \quad \forall v \in W_{h}^{1}, h<\tilde{h}
$$


where $\tilde{h}$ is a sufficiently small fixed number and $C_{1}$ a constant independent on $v$ and $h$.

It should be noted that a similar result can be obtained in the case of curved triangular isoparametric elements which are described in [2] and [3].

Corollary $2:$ Let a part of the boundary $\Gamma_{h}$ be piecewise cubic and the remaining part piecewise quintic. Let $W_{h}^{2}$ be the corresponding $C^{1}$-finite element space which is described in section 1 . Let the boundary $\Gamma$ of $\Omega$ be of class $C^{4}$. Let the assumptions of theorem 1 concerning $S$ and $S_{h}$ be satisfied. Then

$$
\|v\|_{1, \Omega_{h}}^{2} \leqslant C_{1}\left(\int_{S_{h}} v^{2} d s+|v|_{1, \Omega_{h}}^{2}\right) \quad \forall v \in W_{h}^{2}, h<\tilde{h}
$$

where $\tilde{h}$ and $C_{1}$ have the same meaning as in corollary 1.

CoRollary $3:$ Let the assumptions of corollary 2 be satisfied and let $S$ be not a part of a straight line. Then

$$
\|v\|_{2, \Omega_{h}}^{2} \leqslant C_{2}\left(\int_{S_{h}} v^{2} d s+|v|_{2, \Omega_{h}}^{2}\right) \quad \forall v \in W_{h}^{2}, h<\tilde{h}
$$

where $\tilde{h}$ is a sufficiently small fixed number and $C_{2}$ is a constant independent on $v$ and $h$.

\section{APPLICATIONS}

Applications of theorem 1 and its corollary in the case of second order elliptic equations are introduced in [10]. In this section we restrict ourselves to the case of fourth order problems.

Let us consider the following problem of bending of thin elastic plates :

$$
\begin{aligned}
\Delta^{2} u & =f \text { in } \Omega \\
u=g_{0}(s) & , \quad \partial u / \partial v=g_{1}(s) \text { on } \Gamma_{1} \\
u & =g_{2}(s) \text { on } \Gamma_{2} \\
M u & =P(s) \text { on } \Gamma_{2}+\Gamma_{3} \\
N u & =Q(s) \text { on } \Gamma_{3}
\end{aligned}
$$

where $v$ is the outward normal to the boundary $\Gamma=\Gamma_{1}+\Gamma_{2}+\Gamma_{3}$ $\left(\Gamma_{i} \cap \Gamma_{j}=\varnothing\right), f, g_{0}, g_{1}, g_{2}, P, Q$ are sufficiently smooth functions and

$$
\begin{gathered}
M u=\mu \Delta u+(1-\mu) \partial^{2} u / \partial v^{2} \quad(0<\mu<1) \\
N u=-\frac{\partial}{\partial v}(\Delta u)+(1-\mu) \frac{\partial}{\partial s}\left[\frac{\partial^{2} u}{\partial x^{2}} v_{1} v_{2}-\frac{\partial^{2} u}{\partial x \partial y}\left(v_{1}^{2}-v_{2}^{2}\right)-\frac{\partial^{2} u}{\partial y^{2}} v_{1} v_{2}\right]
\end{gathered}
$$


where $\mu$ is a constant, $v_{1}$ and $v_{2}$ are the direction cosines of $v$ and $s$ is the tangential direction with direction cosines $-v_{2}, v_{1}$. We assume that $\Gamma$ is of class $C^{4}$ and that mes $\Gamma_{3}<$ mes $\Gamma$.

Let us define the space

$$
\begin{aligned}
V_{0}=\left\{w \in H^{2}(\Omega): w=0 \text { on } \Gamma_{1}+\Gamma_{2}, \partial w / \partial v=\right. & 0 \text { on } \Gamma_{1} \\
& \text { in the sense of traces }\}
\end{aligned}
$$

and the set

$$
\begin{array}{r}
V_{g}=\left\{w \in H^{2}(\Omega): w=g_{0}(s), \partial w / \partial v=g_{1}(s) \text { on } \Gamma_{1},\right. \\
\left.w=g_{2}(s) \text { on } \Gamma_{2} \text { in the sense of traces }\right\} .
\end{array}
$$

The variational formulation of problem (77)-(81) then reads as follows : Find $u \in V_{g}$ such that

$$
a(u, w)=L(w) \quad \forall w \in V_{0}
$$

where

$$
\begin{gathered}
a(v, w)=\iint_{\Omega}\left[\left(\frac{\partial^{2} w}{\partial x^{2}}+\mu \frac{\partial^{2} w}{\partial y^{2}}\right) \frac{\partial^{2} v}{\partial x^{2}}+\left(\frac{\partial^{2} w}{\partial y^{2}}+\mu \frac{\partial^{2} w}{\partial x^{2}}\right) \frac{\partial^{2} v}{\partial y^{2}}+\right. \\
\left.+2(1-\mu) \frac{\partial^{2} v}{\partial x \partial y} \frac{\partial^{2} w}{\partial x \partial y}\right] d x d y \\
L(w)=L^{\Omega}(w)+L^{\Gamma}(w) . \\
L^{\Omega}(w)=\iint_{\Omega} f w d x d y \\
L^{\Gamma}(w)=\int_{\Gamma_{2}+\Gamma_{3}} P \frac{\partial w}{\partial v} d s+\int_{\Gamma_{3}} Q w d s .
\end{gathered}
$$

We shall solve the problem (86) by the finite element method. Let $W_{h}^{2}$ be the $C^{1}$-finite element space introduced in section 1 . Then, according to (23), $\Gamma_{h 1}+\Gamma_{h 3}$ is piecewise cubic and $\Gamma_{h 2}$ precewise quintic. Let $V_{0 h}$ be a subspace of $W_{n}^{2}$ defined by

$$
V_{0 h}=\left\{w \in W_{h}^{2}: w=0 \text { on } \Gamma_{h 1}+\Gamma_{h 2}, \partial w / \partial v=0 \text { on } \Gamma_{h 1}\right\}
$$

where $v_{h}$ is the outward normal to $\Gamma_{h}$. Finally, let $V_{g_{h}}$ be the subset of $W_{h}^{2}$ consisting of those functions which satisfy the following at the nodal points $P_{l}$ lying on $\Gamma_{h}$ : boundary conditions (78) and (79) and all consequences of these conditions containing at most second order derivatives. (The reason : according to the definition of $W_{h}^{2}$, the derivatives $D^{\alpha} w\left(P_{\imath}\right), \mid \alpha^{2} \leqslant 2$ are all 
parameters prescribed at $P_{1} \in \Gamma_{h}$.) E.g., if $P_{\imath} \in \Gamma_{h 2}$ then every function $w \in W_{h}^{2}$ satisfies the following conditions at $P_{\imath}$ :

$$
\begin{gathered}
w\left(P_{\imath}\right)=g_{2}\left(s_{\imath}\right), \varphi^{\prime}\left(s_{\imath}\right) \frac{\partial w}{\partial x}\left(P_{\imath}\right)+\psi^{\prime}\left(s_{\imath}\right) \frac{\partial w}{\partial y}\left(P_{\imath}\right)=g_{2}^{\prime}\left(s_{\imath}\right), \\
g_{2}^{\prime \prime}\left(s_{\imath}\right)=\left[\varphi^{\prime}\left(s_{\imath}\right)\right]^{2} \frac{\partial^{2} w}{\partial x^{2}}\left(P_{\imath}\right)+2 \varphi^{\prime}\left(s_{\imath}\right) \psi^{\prime}\left(s_{\iota}\right) \frac{\partial^{2} w}{\partial x \partial y}\left(P_{\imath}\right)+ \\
+\left[\psi^{\prime}\left(s_{\iota}\right)\right]^{2} \frac{\partial^{2} w}{\partial y^{2}}\left(P_{\iota}\right)+\varphi^{\prime \prime}\left(s_{\imath}\right) \frac{\partial w}{\partial x}\left(P_{\imath}\right)+\psi^{\prime \prime}\left(s_{\imath}\right) \frac{\partial w}{\partial y}\left(P_{\imath}\right) .
\end{gathered}
$$

Now we can formulate the discrete problem corresponding to problem (86) : find $\tilde{u}_{h} \in V_{g h}$ such that

$$
\tilde{a}_{h}\left(\tilde{u}_{h}, w\right)=\tilde{L}_{h}(w) \quad \forall w \in V_{0 h}
$$

where $\tilde{a}_{h}(v, w)$ is defined by an expression similar to the expression appearing on the right-hand side of (87) - only $\Omega$ is substituted by $\Omega_{h}$. The linear form $\tilde{L}_{h}(w)$ is defined by the relation analogous to $(88)$ and

$$
\begin{gathered}
\tilde{L}_{h}^{\Omega}(w)=\iint_{\Omega_{h}} \tilde{f} w d x d y \\
\tilde{L}_{h}^{\Gamma}(w)=\int_{\Gamma_{h 2}+\Gamma_{h 3}} P_{h} \frac{\partial w}{\partial v_{h}} d s+\int_{\Gamma_{h 3}} Q_{h} w d s .
\end{gathered}
$$

The symbol $\tilde{f}$ denotes a contınuous extension of the function $f$ to a domain $\widetilde{\Omega} \supset \Omega_{h}(h<\widetilde{h})$ and $P_{h}, Q_{h}$ are functions obtained by "transferring " the functions $P, Q$ from $\Gamma$ onto $\Gamma_{h}$ (we explain it in the case of the function $Q$ ) : let $c\left(P_{2}, P_{3}\right)$ be an arc lying on $\Gamma_{3}, P_{2}$ and $P_{3}$ being 1 ts end points denoted in a local notation. Let $c_{h}\left(P_{2}, P_{3}\right) \subset \Gamma_{h 3}$ be the approximation of $c\left(P_{2}, P_{3}\right)$. The parametric representations of $c\left(P_{2}, P_{3}\right)$ and $c_{h}\left(P_{2}, P_{3}\right)$ are expressed by (6) and (12), respectively. To every point $t \in[0,1]$ there exists just one point

$$
(x, y)=\left(\varphi^{*}(t), \psi^{*}(t)\right) \in c_{h}\left(P_{2}, P_{3}\right)
$$

and just one point $(\bar{\varphi}(t), \bar{\psi}(t)) \in c\left(P_{2}, P_{3}\right)$. Thus at every point (95) we can define the function $Q_{h}(x, y)$ by the relation

$$
Q_{h}(x, y)=Q(\bar{\varphi}(t), \bar{\psi}(t))
$$

Then, according to the definition of the line integral, we have

$$
\int_{c_{h}} Q_{h} w d s=\int_{0}^{1} Q(\bar{\varphi}(t), \bar{\psi}(t)) w\left(\varphi^{*}(t), \psi^{*}(t)\right) \rho^{*}(t) d t
$$

where $\rho^{*}(t)$ is given by $(67)$. 
Let us restrict to the case that $f$ is a constant. Then $\tilde{f}=f$ and from the theoretical point of view it is possible to solve problem (92) and not to use numerical integration. As the applications of theorems 1 and 2 are the same in both cases with and without numerical integration we restrict our considerations to problem (92). (Details concerning the use of numerical integration can be found in [4].)

It follows from (10), (11) and from the definition of the space $V_{0 h}$ and the set $V_{g h}$ that it holds

$$
v, w \in V_{g h} \Rightarrow v-w \in V_{0 h} .
$$

Using this implication it is easy to prove the following theorem (the proof is omitted because it is only a modification of the proofs of similar theorems from [2] and [3]) :

THEOREM 3 : Let a family of discrete problems (92) be given. Let there exist a constant $\gamma>0$ independent on $h$ such that we have for $h<\tilde{h}$

$$
\gamma\|v\|_{2, \Omega_{h}}^{2} \leqslant \tilde{a}_{h}(v, v) \quad \forall v \in V_{0 h} .
$$

Then for every $h<\tilde{h}$ problem (92) has a unique solution $\tilde{u}_{h}$ and it holds

$$
\left\|\tilde{u}-\tilde{u}_{h}\right\|_{2, \Omega_{h}} \leqslant C\left[\inf _{v \in V_{g h}}\|\tilde{u}-v\|_{2, \Omega_{h}}+\sup _{w \in V_{0 h}} \frac{\left|\tilde{a}_{h}(\tilde{u}, w)-\tilde{L}_{h}(w)\right|}{\|w\|_{2, \Omega_{h}}}\right]
$$

where $\tilde{u}$ is an arbitrary function in $\tilde{H}^{2}(\tilde{\Omega})$ and $C$ is a constant independent on $\tilde{u}$ and $h$.

In what follows $\tilde{u}$ will denote a continuous extension of the exact solution of problem (77)-(81) to the domain $\widetilde{\Omega} \supset \Omega_{h}(h<\tilde{h})$. We shall use theorem 3 for estimating $\left\|\tilde{u}-\tilde{u}_{h}\right\|_{2, \Omega_{h}}$.

First we establish the validity of inequality (99) which expresses the uniform $V_{0 h}$-ellipticity of the bilinear forms $\tilde{a}_{h}(v, w)(h<\tilde{h})$. It is easy to see that

$$
\tilde{a}_{h}(v, v) \geqslant(1-\mu)|v|_{2, \Omega_{h}}^{2} \quad \forall v \in W_{h}^{2}, \quad \forall h .
$$

If mes $\Gamma_{1}>0$ and $\Gamma_{1}$ is not a part of a straight line we use corollary 3 and (101) for arbitrary $v \in V_{0 h}$. We obtain inequality (99) with $\gamma=(1-\mu) / C_{2}$. If $\Gamma_{1}$ is a part of a straight line we use the inequality

$$
|v|_{2, \Omega_{h}}^{2} \geqslant \frac{1}{2}\left(|\partial v / \partial x|_{1, \Omega_{h}}^{2}+|\partial v / \partial y|_{1, \Omega_{h}}^{2}\right) .
$$

Modifying some ideas of the proof of theorems 1 and 2 it is not difficult to prove that

$$
C_{1}\left(|\partial v / \partial x|_{1, \Omega_{h}}+|\partial v / \partial y|_{1, \Omega_{h}}^{2}\right) \geqslant|v|_{1, \Omega_{h}}^{2} \quad \forall v \in V_{0 h}
$$


where $h<\tilde{h}$ and $C_{1}$ is the constant from corollaries 1 and 2. Using then corollary 2 we obtain inequality (99) with

$$
\gamma=\frac{1}{2}(1-\mu) \min \left(1,1 /\left(2 C_{1}^{2}\right)\right) .
$$

If mes $\Gamma_{1}=0$ then mes $\Gamma_{2}=$ mes $\Gamma-$ mes $\Gamma_{3}>0$. In this case we assume that $\Gamma_{2}$ is not a part of a straight line (a necessary condition for the uniqueness of the solution of problem (77)-(81)). Using (101) and corollary 3 for arbitrary $v \in V_{0 h}$ we obtain inequality (99) with $\gamma=(1-\mu) / C_{2}$.

In estimating the first term on the right-hand side of $(100)$ we shall use $[9$, theorem 5] and a similar theorem for Bell's elements [1, p. 819]. In accordance with assumptions of these theorems we shall assume that $\tilde{u} \in H^{5}(\tilde{\Omega})$. Let $w_{I}$ be the function from $W_{h}^{2}$ which interpolates $\tilde{u}$ (i.e. the parameters uniquely determining $w_{I}$ are the function values and derivatives of $\tilde{u}$ at the corresponding nodal points). Then $w_{I} \in V_{g h}$ and we have

$$
\inf _{v \in V_{g h}}\|\tilde{u}-v\|_{2, \Omega_{h}} \leqslant\left\|\tilde{u}-w_{I}\right\|_{2, \Omega_{h}} \leqslant C h^{3}\|\tilde{u}\|_{5, \Omega_{h}} .
$$

It remains to prove that the second term on the right-hand side of $(100)$ is $O\left(h^{3}\right)$. The assumption $\tilde{u} \in H^{5}(\tilde{\Omega})$ allows to use Green's theorem and we can establish

$$
\begin{aligned}
\left|\tilde{a}_{h}(\tilde{u}, w)-\tilde{L}_{h}(w)\right| \leqslant\left|\iint_{\Omega_{h}}\left(\Delta^{2} \tilde{u}-f\right) w d x d y\right|+ \\
\quad+\left|\int_{\Gamma_{h 2}+\Gamma_{h 3}}\left(M_{h} \tilde{u}-P_{h}\right) \frac{\partial w}{\partial v_{h}} d s\right|+\left|\int_{\Gamma_{h 3}}\left(N_{h} \tilde{u}-Q_{h}\right) w d s\right|
\end{aligned}
$$

where the operators $M_{h}$ and $N_{h}$ are defined by relations similar to (82) and (83), respectively. (The only change is that we write $v_{h}, v_{h 1}, v_{h 2}, s_{h}$ instead of $v, v_{1}$, $v_{2}$, s.) As $\Gamma$ is approximated by $\Gamma_{h}$ at least with accuracy $0\left(h^{4}\right)$ and $\Delta^{2} u-f=0$ in $\Omega$ it is easy to find that

$$
\left|\iint_{\Omega_{h}} w\left(\Delta^{2} \tilde{u}-f\right) d x d y\right| \leqslant\|w\|_{2, \Omega_{h}} O\left(h^{6}\right) .
$$

Let us denote for simplicity $\sigma_{1}=M_{h} \widetilde{u}-P_{h}, \sigma_{2}=N_{h} \widetilde{u}-Q_{h}$. Using the Cauchy inequality and the trace theorem we can write

$$
\begin{gathered}
\left|\int_{\Gamma_{h 2}+\Gamma_{h 3}} \sigma_{1} \frac{\partial w}{\partial v_{h}} d s\right| \leqslant C \sqrt{\operatorname{mes} \Gamma_{h}} \max _{\Gamma_{h 2}+\Gamma_{h 3}}\left|\sigma_{1}\right| \cdot\|w\|_{2, \Omega_{h}}, \\
\left|\int_{\Gamma_{h 3}} \sigma_{2} w d s\right| \leqslant C \sqrt{\operatorname{mes} \Gamma_{h}} \max _{\Gamma_{h 3}}\left|\sigma_{2}\right| \cdot\|w\|_{2, \Omega_{h}} .
\end{gathered}
$$


It follows from the proof of the trace theorem (see [6, p. 15-16]) and from lemma 2 (or remark 2, part (i)) that the constant $C$ appearing in (105) and (106) is independent on $h$.

According to the Taylor formula and lemma 2 (or remark 2) we have

$$
\begin{aligned}
\Delta \tilde{u}\left(\varphi^{*}(t), \psi^{*}(t)\right) & =\Delta u(\bar{\varphi}(t), \bar{\psi}(t))+O\left(h_{T}^{4}\right), \\
\frac{\partial^{2} \tilde{u}}{\partial v_{h}^{2}}\left(\varphi^{*}(t), \psi^{*}(t)\right) & =\frac{\partial^{2} u}{\partial v^{2}}(\bar{\varphi}(t), \bar{\psi}(t))+0\left(h_{T}^{3}\right) .
\end{aligned}
$$

Thus

As

$$
\left.M_{h} \tilde{u}\right|_{c_{h}}=\left.M u\right|_{c}+O\left(h_{T}^{3}\right)
$$

$$
\begin{gathered}
\left.M u\right|_{c}=P(\bar{\varphi}(t), \bar{\psi}(t)), \\
\left.P_{h}\right|_{c_{h}} \equiv P_{h}\left(\varphi^{*}(t), \psi^{*}(t)\right)=P(\bar{\varphi}(t), \bar{\psi}(t))
\end{gathered}
$$

we obtain from (107)

$$
\max _{c_{h}}\left|M_{h} \tilde{u}-P_{h}\right|=0\left(h_{T}^{3}\right), c_{h} \subset \Gamma_{h 2}+\Gamma_{h 3} .
$$

As mes $\Gamma_{h} \leqslant 2$ mes $\Gamma$ relations (105) and (108) imply

$$
\left|\int_{\Gamma_{h 2}+\Gamma_{h 3}}\left(M_{h} \tilde{u}-P_{h}\right) \frac{\partial w}{\partial v_{h}} d s\right| \leqslant C h^{3}\|w\|_{2, \Omega_{h}} .
$$

Similarly we obtain

$$
\left|\int_{\Gamma_{h 3}}\left(N_{h} \tilde{u}-Q_{h}\right) w d s\right| \leqslant C h^{3}\|w\|_{2, \Omega_{h}} .
$$

Theorem 3 and estimates (102), (103), (104), (109), (110) give the final result : the solution $\tilde{u}_{h}$ of the discrete problem (92) exists and is unique for sufficiently small $h$. If $\tilde{u} \in H^{5}(\tilde{\Omega})$ then $\left\|\tilde{u}-\tilde{u}_{h}\right\|_{2, \Omega_{h}}=O\left(h^{3}\right)$.

Remark $4:$ Let $V_{0}=\left\{v \in H^{1}(\Omega): v=0\right.$ on $\Gamma_{1}$ in the sense of traces $\}$. The minimum value of $K_{1}(\Omega)$ in Friedrichs' inequality

$$
|v|_{0, \Omega}^{2} \leqslant K_{1}(\Omega)|v|_{1, \Omega}^{2} \quad \forall v \in V_{0}
$$

is equal to $\lambda_{1}^{-1}$ where $\lambda_{1}$ is the first eigenvalue of the problem

$$
\Delta u+\lambda u=0 \text { in } \Omega, u=0 \text { on } \Gamma_{1}, \quad \partial u / \partial v=0 \text { on } \Gamma-\Gamma_{1} .
$$

It holds

$$
\lambda_{1}=\min _{v \in V_{0}} \frac{|v|_{1, \Omega}^{2}}{|v|_{0, \Omega}^{2}}=\frac{\left|u_{1}\right|_{1, \Omega}^{2}}{\left|u_{1}\right|_{0, \Omega}^{2}}
$$


where $u_{1}$ is the first eigenfunction of problem (112). Let us assume that $\Gamma$ is of class $C^{n+1}$ and let us define the finite element approximation of $\lambda_{1}$ by

$$
\lambda_{1 h}=\inf _{v \in V_{0 h}} \frac{|v|_{1, \Omega_{h}}^{2}}{|v|_{0, \Omega_{h}}^{2}} .
$$

Considerations introduced in the proof of theorems 1 and 2 imply $\lambda_{1 h} \geqslant \lambda_{1}\left(1+O\left(h^{n}\right)\right)$. Thus

$$
\lim _{h \rightarrow 0} \lambda_{1 h} \geqslant \lambda_{1}
$$

In the case $n \geqslant 2, u_{1} \in H^{n+1}(\Omega)$ it can be proved that

$$
\lim _{h \rightarrow 0} \lambda_{1 h}=\lambda_{\mathbf{i}} \text {. }
$$

Let $w_{I h} \in V_{0 h}$ be the interpolate of the extension $\tilde{u}_{1} \in H^{n+1}(\widetilde{\Omega})$ of $u_{1}$. It is not difficult to find that

$$
\lim _{h \rightarrow 0}\left|w_{I h}\right|_{i, \Omega_{h}}^{2}=\left|u_{1}\right|_{i, \Omega}^{2} \quad(i=0,1)
$$

where the rate of convergence is $O\left(h^{n-i}\right)$. We have

$$
\left|w_{I h}\right|_{1, \Omega_{h}}^{2} /\left|w_{I h}\right|_{0, \Omega_{h}}^{2} \geqslant \lambda_{1 h} .
$$

Letting $h \rightarrow 0$ we obtain from (117), (118) and (113)

$$
\lambda_{1} \geqslant \lim _{h \rightarrow 0} \lambda_{1 h}
$$

Inequalities (115) and (119) imply the desired result (116).

\section{REFERENCES}

[1] J. H. Bramble, M. Zlámal, Triangular elements in the finite element method. Math. Comp. 24 (1970), 809-820.

[2] P. G. Ciarlet, P. A. RAviart, The combined effect of curved boundaries and numerical integration in isoparametric finite element methods. In : The Mathematical Foundations of the Finite Element Method with Applications to Partial Differential Equations (A. K. Aziz, Editor), Academic Press, New York, 1972, pp. 409. 474.

[3] P. G. Ciarlet, The Finite Element Method for Elliptic Problems. North-Holland, Amsterdam, 1978.

[4] J. Ȟ̌EBÍČEK, A numerical analysis of a general biharmonic problem by the finite element method. (To appear.) 
A ŽENÍŠEK

[5] L MANSFIELD, Approximation of the boundary in the finite element solution of fourth order problems SIAM J Numer Anal 15(1978), 568-579

[6] J NeČAS, Les methodes directes en theorle des equations elliptiques Academia, Prague, 1967

[7] M Zlamal, Curved elements in the finite element method I SIAM J Numer Anal 10 (1973), 229-240

[8] M Zlamal, Curved elements in the finte element method II SIAM J Numer Anal 11 (1974), 347-362

[9] A ŽENišEK, Curved triangular finite $C^{m}$-elements Apl Mat 23 (1978), 346-377

[10] A ŽENIŠEK, Nonhomogeneous boundary condittons and curved triangular finite elements Apl Mat 26(1981), 121-141 